\title{
A Case Analysis of Turner's syndrome for Neonate
}

\author{
Xueping Zhu*, Xiaoqian Chen, Zhihui Xiao and Xiaoyun Zhao \\ Department of Neonatology, Children's Hospital of Soochow University, China \\ *Corresponding author: Xueping Zhu, Department of Neonatology, Children's Hospital of Soochow University, Suzhou, Jiangsu China 215000, China
}

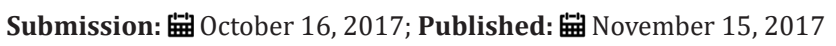

\begin{abstract}
Background: Turner 's syndrome (TS) is the only monosomic syndrome in humans with affected individuals who may survive.

Methods: we reported an 8-day old female infant who was diagnosed as TS. Detailed information regarding as clinical features, diagnosis, management, and outcome are provided.

Results: The main clinical features were legathy, purple face, weak cry and shortness of breath, other manifestations including edema in the back of the neck, dyspnea with obvious retractions, rales in both lungs, edema in lower legs, feet and joints without pitting, and poorly developed toe nails. Chromosomal analysis of peripheral blood shows a karyotype of $45, \mathrm{XO}$ and thus the diagnosis in the newborn stage of life, concurrently suffering from lung infection and congenital heart disease.

Conclusion: As a common chromosomal disorder, TS has the special clinical features, diagnosed according to the Chromosomal analysis of peripheral blood, and can be recognized at neonatal stage. If we can diagnose early, the prognosis of patients will be better. A full understanding of the prognosis of the disorder and effective communication with patients and their family are crucial to improve the life quality of TS sufferers.

Keywords: Turner's syndrome; Clinical features; Diagnosis; Prognosis; neonate
\end{abstract}

\section{Introduction}

Turner's Syndrome (TS) has been recognized as a genetic disorder in females since 1959, so named since the first report of Turner in 1938 [1], The diagnosis of TS is usually delayed, 4\% of which after age of 7 , seen for their short stature [2] and 30\% diagnosed in adolescence because of primary amenorrhea. Rarely, the patient has been diagnosed in neonatal period. We report an 8-day female infant who was diagnosed as TS. We thus provide detailed clinical information and discuss diagnosis and treatment of this uncommon case.

\section{Case Report}

An 8-day old female infant, admitted to our hospital secondary to shortness of breath.

I. Birth history: G1P1, full term, delivered by C-section on April 1, 2009 in Taicang No. 1 Hospital, Suzhou. MSAF: III; Apgar Scores: $9^{1} 9^{5}$; BW: $3.36 \mathrm{~kg}$, with notable purple face, foaming mouth and shortness of breath.

II. Parental history: 24-year-old father and 23-year-old mother, healthy workers for a down wear factory. No family history of similar disorders or other infectious or genetic diseases.

III. Physical examination: T: $36.8^{\circ} \mathrm{C}, \mathrm{R}: 61, \mathrm{P}: 140$, weight: $3.27 \mathrm{~kg}$, body length: $49 \mathrm{~cm}$, head circumference: $35 \mathrm{~cm}$, chest circumference: $34 \mathrm{~cm}$, with features consistent with a full-term infant, normal nasal bridge, eye space and location of ears, without droopy eyelid or edema. Other findings included legathy, purple face, weak cry and shortness of breath; soft anterior fontanel (about $1.5 \times 1.5 \mathrm{~cm}$ ), equal round pupils and normal sensitivity to light, no neck stiffness or webbing but edema in the back of the neck; dyspnea with obvious retractions, coarse breath sounds with rales in both lungs; regular heart beats, HR: $140 \mathrm{bpm}$; soft abdomen with liver palpated $1.5 \mathrm{~cm}$ below the costal margin of normal texture; normal tension in all 4 extremities but edema in lower legs, feet and joints without pitting; normal development of upper limbs including elbows but poorly developed toe nails.

IV. Laboratory Data: Normal CBC, normal liver and renal function tests, normal electrolytes but low blood glucose, as well as normal ABG on admission to the hospital; abnormally high activated partial thromboplastin time (APTT, 42 seconds), low fibrinogen of $1.9 \mathrm{~g} / \mathrm{L}$; free T3, 2.15pg/ml (lower); free T4, 1.00ng/ml; TSH, 1.4iu/ $\mathrm{ml}$; total T3, $0.47 \mathrm{ng} / \mathrm{ml}$ (lower); total T4, $101.89 \mathrm{ng} / \mathrm{ml}$; low levels of both total protein and albumins. Chest X-ray suggests pneumonia, right pleural effusion with elevation of the diaphragm on the right. Color Doppler ultrasound of the heart demonstrates patent ductus arteriosus, patent foramen ovale, pulmonary hypertension and right pleural effusion of $30 \times 26 \mathrm{~mm}$ in volume. Blood cultures are negative; bacterial culture of sputum returns Streptococus viridans $(+++)$, Staphylococcus epidermidis $(+++)$ and rare Gram negative bacilli. Chromosomal analysis of peripheral blood shows a karyotype of $45, \mathrm{XO}$. 
V. Hospital Course: Upon hospitalization, the infant was given Sulperazon for infection, Mucosolvan for reduction of phlegm, Cedilandi and Furosemide for dieresis; CPAP for enhanced oxygenation and other medications for improved circulation. Other treatment included strict intravenous fluid input and TPN support. Three days after hospitalization, ABG shows PH7.2, PaO2 48.2mmHg, PaCO2 62.7mmHg, BE-B -4.2mmol/l and Glu 8.2mmol/l.

VI. Diagnosis: Turner 's syndrome; neonatal pneumonia; pleural effusion and congenital heart disease. Condition on discharge: The infant was not fully recovered from pneumonia or pleural effusion, and a mask was needed for oxygen, but treatment was discontinued upon request of parents.

\section{Discussion}

Turner's Syndrome (TS) has been recognized as a genetic disorder in females since 1959, so named since the first report of Turner in 1938 [1]. The ovarian tissue of the affected is replaced by streaks of connective tissue leading to absence of female sex hormones, which in turn leads to poor development of secondary sexual characteristics and primary amenorrhea. TS is the only monosomic syndrome in humans with affected individuals who may survive. Typical karyotype of TS is 45 , XO, originating from complete or partial missing of the $\mathrm{X}$ chromosome. The clinical manifestations of TS vary, but mainly consist of short stature, widely spaced nipples, evagination of forearms, congenital lymphedema, lack of pubertal development, webbed neck, cardiovascular malformations, damaged glucose tolerance, disorders of the thyroid glands and hearing loss [3-8]. TS occurs in 1 out of 25003000 live female newborns [9]. Spontaneous abortion may occur in $99 \%$ of fetuses with TS of 45 , X karyotype, $10 \%$ of which in the first three months of pregnancy [10]. However, maternal age is not a risk factor for TS [11].

Fifty percent of karyotypes in TS are 45 , XO or non-mosaic types. Others include deletion of the short or the long arm, ring X, isochromosome of one arm, and mosaicism, where some of the cells in the body have a Turner syndrome chromosome complement and other cells in the body have a different chromosome complement, either normal (karyotype 45,X/46,XX) or abnormal (karyotype $45, X / 47, X X X)$. Many reports have shown that clinical symptoms in mosaic hosts are mild, but the relationship between different karyotypes and cognitive functions is not clear.

The diagnosis of TS is usually delayed, $4 \%$ of which after age of 7 , seen for their short stature [2] and 30\% diagnosed in adolescence because of primary amenorrhea. The reported case was admitted 8 days after birth for respiratory disorders. Findings of edema in lower limbs, as well as in the back of the neck, poor development of toe nails led to further chromosomal study of peripheral blood showing 45 , XO, the typical karyotype of TS and thus the diagnosis in the newborn stage of life.

Treatment of the TS affected includes monitoring and treating congenital heart disease and the use of growth hormone to normalize growth curves (as early as 1-2 years old) in childhood and adolescence, estrogen replacement to promote the development of sex organs and to maintain bone density (usually initiation of estrogen supplement before age 13) [12]. The height of untreated TS individuals averages $140 \mathrm{~cm}$ in adulthood, which may reach an average of $150 \mathrm{~cm}$ in the treated group, with both growth hormone and estrogen $[13,14]$. Treatment with growth hormone usually lasts up to the bone age of 14 while estrogen replacement is needed for the entire life time [15].

Management of TS during adulthood mainly involves the maintenance of sexual organ development and reproductive potential. 2-5\% of TS patients of typical karyotype and those of 46, $\mathrm{XX} / 45$, X mosaicism may experience spontaneous menstruation and pregnancy. In addition, treating physicians should not ignore the psychosocial challenges TS sufferers may have from their short stature, sterility and poor development of sexual characteristics. Treatment of our patient analyzed above discontinued after 2 weeks since the lack of confidence from parents as well as economical reasons. A full understanding of the prognosis of the disorder and effective communication with patients and their family are crucial to improve the life quality of TS sufferers.

\section{References}

1. Turner HH (1983) A syndrome of infantilism,congenital webbed neck, and cubitus valgus. Endocrinology 23: 566-574.

2. Jones KL, Smith DW (2006) Smith's Recognizable Patterns of Human Malformation ( $6^{\text {th }}$ edn), Elsevier Sauders, Philadelphia, USA, pp. 76-81.

3. Gravholt CH (2005) Clinical practice in Turner syndrome. Nat Clin Pract Endocrinol Metab 1(1): 41-52.

4. Bondy CA, Bakalov VK (2006) Investigation of cardiac status and bone mineral density in Turner syndrome. Growth Horm IGF Res 16: s103-s108.

5. Dhooge IJ (2005) Otologic disease in turner syndrome. Otol Neurotol 26(2): 145-150.

6. Gungor N (200) High frequency hearing loss in Ullrich-Turner Syndrome. Eur J Pediatr 159(10): 740-744.

7. Zinn AR (1998) Evidence for a Turner Syndrome locus or loci at xp11.2-p22.1. Am J Hum Genet 63(6): 1757-1766.

8. Cassidy SB, Allanson JE (2005) Management of Genetic Syndromes. $\left(2^{\text {nd }}\right.$ edn), NJ John Wiley \& sons, Hoboken.

9. Menasha J, Levy B, Hirschhorn K, Kardon NB (2005) Incidence and spectrum of chromosome abnormalities in spontaneous abortions: new insights from a 12-year study. Genet Med 7: 251-263.

10. Gardner RJ, Sutherland GR (2004) Chromosome abnormalities and Genetic Counseling. ( $3^{\text {rd }}$ edn), NY Oxford University Press, NewYork, USA, pp. 199-200.

11. Savendahl, Davenport ML (2000) Delayed diagnoses of Turner Syndrome: proposed guidelines for change. J Pediatr (3): 455-459.

12. Rosenfield RL, Devine N, Hunold JJ (2005) Salutary effects of combiong early very low-dose systemic estradiol with growth hormone therapy in girls with Turner syndrome. J Clin Endocrinol Metab 90: 6424-6430.

13. Stephure DK (2005) Canadian Growth hormone Advisory committee. Impact of growth hormone supplementation on adult height in Turner syndrome: results of the canadian randonized controlled trial. J Clin Endocrinol Metab 90: 3360-3366.

14. Ping-Yi Hsu, Yi-ching Tung, Wen-Yu Tsai (2008) Effect of Growth Hormone Therapy on Adult height of children with Turner syndrome. J Forms Med Assoc 107(9): 704-709.

15. Hanton L, Axelrod L, Bakalov V (2003) The importance of estrogen replacement in young women with Turner syndrome. J Women's Health 12: 971-977. 